\title{
Effect of linezolid on hematological and oxidative parameters in rats
}

\author{
Tianlin Wang, Daihong Guo, Xianzhe Dong and Lihua Mu
}

Effect of linezolid is associated with hematocytopenia. A possible relationship between oxidative stress and hematologic toxicity has also been discussed. This present study was performed to determine the effect of oral administration of linezolid on hematological and oxidative parameters in rats. Rats were divided into five groups of 20 each. Drugs were given for a 15-day period. Control rats were vehicle treated. In the second and third groups, linezolid $\left(50\right.$ and $125 \mathrm{mg} \mathrm{kg}^{-1}$ ) was given to rats twice a day ( 100 and $250 \mathrm{mg} \mathrm{kg}^{-1}$ per day) orally by gavage. The fourth group was given diet supplement that contained $250 \mathrm{mg} \mathrm{kg}^{-1}$ Vitamin $\mathrm{E}$. The fifth group was given $125 \mathrm{mg} \mathrm{kg}^{-1}$ linezolid twice a day along with Vitamin $\mathrm{E}$ supplement. Blood cell counts (red blood cell (RBC), white blood cell (WBC) and platelet) were measured every 5 days. Enzymatic antioxidant activities, lipid peroxidation and free radical production were performed at the end of 15 th day. The results showed that linezolid induces a decrease of WBC, RBC and platelet numbers in rat blood and enzymatic activities of superoxide dismutase and catalase in rat serum in a dose-dependent manner. While the content of malondialdehyde and nitric oxide was significantly increased in rats serum compared with the control group, Vitamin E supplement could partially reverse the above changes without affecting the control group. The results suggest that linezolid has effect on decreasing hemocyte level in rats, and oxidative stress may be one of the major causative factors. Antioxidant may prove to be a useful therapeutic option in the reversal of hematologic effects induced by linezolid.

The Journal of Antibiotics (2014) 67, 433-437; doi:10.1038/ja.2014.21; published online 2 April 2014

Keywords: free radical; hematological effect; linezolid; lipid peroxidation; oxidative stress

\section{INTRODUCTION}

Severe hematological adverse events are often seen in patients with antimicrobial agents treatment. Chloramphenicol is the most studied object. ${ }^{1,2} \quad \beta$-Lactams, quinolones, cephalosporins, imethoprimsulfamethoxazole and vancomycin are also included in recent years. ${ }^{3-5}$ However, the pathogenesis of hematological adverse events is poorly understood. Actual researches were merely restricted to direct toxicity to hematopoietic cells (Classic myelosuppression observed with chloramphenicol is thought to occur through direct toxicity of mitochondria, thus inhibiting protein synthesis). ${ }^{6}$ Immune mechanisms may also have a role due to the production of druginduced antibody, which can cause destruction of the specific hematopoietic cell line. With drug treatment, hemolytic anemia occurs through induction of an autoimmune response to the combination of drugs with cell membrane proteins, resulting in formation of a complex of drug, antibody and cell membrane or a hapten. The autoimmune complex results in hemolysis of blood cells. ${ }^{7}$

Linezolid, a prototype member of the oxazolidinones, has been known for years as a valuable acquisition in the antibiotic armentarium because of its excellent activity against drug-resistant, Grampositive pathogens and its favorable pharmacokinetics. With increased use of linezolid, reports of dose-limiting adverse reactions have been published, inducing mild gastrointestinal adverse events (nausea, vomiting and diarrhea), headaches and fever. ${ }^{8,9}$ However, linezolid use, particularly prolonged courses ( $>14$ days), has been associated with hematological adverse events, anemia and thrombocytopenia, being the most common. ${ }^{10-12}$

Oxidative stress was made in the understanding for mechanisms of hematologic toxicity of various causes. Studies show that oxidative stress has a role in the ethiopathogenesis of thrombocytopenia. Soundravally et al. ${ }^{13}$ reported that thrombocytopenia of dengue infection was associated with the extent of lipid peroxidation. Erel et al. ${ }^{14}$ also indicated that oxidative damage of thrombocytes might be important in the ethiopathogenesis of thrombocytopenia occurring in malaria. However, besides the previous reports available on this aspect, whether oxidative stress played an important role in thrombocytopenia of linezolid is not well documented. Therefore, the present study is undertaken to investigate the relationship between hematological toxicity and oxidative stress occurring in rats with linezolid.

\section{MATERIALS AND METHODS}

Subjects

In the present study, linezolid was obtained as a commercial infusion solution (Zyvoxid; 600 mg per 300 ml; Pfizer, Fresenius kabi norge AS, Norway, Denmark). The diet supplement with vitamin contained $250 \mathrm{mg} \mathrm{kg}^{-1}$ Vitamin E. 
Male Sprague-Dawley rats ( $n=100,180-200 \mathrm{~g}$ body weight) were used in the experiments, which were provided by the Experimental Animal Center of Chinese PLA General Hospital. They were housed four per cage, the room temperature was maintained at $22 \pm 0.5^{\circ} \mathrm{C}$ with a controlled 12 light-dark cycle. Rats were fed ad libitum water and food. All procedures were in accordance with the Regulation of Experimental Animal Administration issued by the State Committee of Science and Technology of the People's Republic of China. The experiments were carried out under the approval of the Committee of Experimental Animal Administration of the Hospital. Rats were divided into five groups with 20 animals in each group: one control group received saline, and four experimental groups were as follows: linezolid 100 and $250 \mathrm{mg} \mathrm{kg}^{-1}$ per day groups; Vitamin E group; Vitamin E + linezolid $250 \mathrm{mg} \mathrm{kg}^{-1}$ per day group. Saline or linezolid was administered intragastrically into rats twice a day (b.i.d.).

A volume of $20 \mu \mathrm{l}$ venous blood samples was collected after an overnight fasting at days $0,5,10$ and 15 , and were injected directly into the automated cell counter immediately (COULTER LH 750 Hematology Analyzer, Beckman Coulter, Brea, CA, USA) for whole blood cell counting. All animals were killed at day 15 , and whole blood was centrifuged ( 3000 r.p.m., $10 \mathrm{~min}, 4{ }^{\circ} \mathrm{C}$ ) to obtain serum, and prepared rapidly for lipid peroxidation and free radical and enzymatic analysis.

\section{Estimation of lipid peroxidation and free radical in serum}

As lipid peroxidation production, malondialdehyde (MDA) content in serum was determined by the thiobarbituric acid method, ${ }^{15}$ monitoring the change of absorbance at $532 \mathrm{~nm}$ with a spectrophotometer (UV-2100, Shanghai Jinhua Technology Instrument Co., Ltd, Shanghai, China). The nitric oxide (NO) content was expressed by the content of NO metabolites (nitrite and nitrate). Nitrate was reduced first to nitrite by the action of nitrate reductase, then the reaction was initiated by the addition of the Griess reagent, and absorbance of the mixture at $550 \mathrm{~nm}$ was determined. The test kits for measuring the contents of MDA (A003-1) and NO (A012) were purchased from Nanjing Jiancheng Institute of Bioengineering (Nanjing, Jiangsu, China), and was performed according to the instructions of the manufacturer. The content of MDA and NO in serum was expressed as $\mathrm{nmolg}^{-1}$ protein and $\mu \mathrm{molg}^{-1}$ protein, respectively.

\section{Determination of enzymatic antioxidant activities in serum}

For the enzyme assays, superoxide dismutase (SOD), glutathione-peroxidase (GSH-Px) and catalase (CAT) activities in serum were determined with the spectrophotometer at $550 \mathrm{~nm}, 412 \mathrm{~nm}$ and $405 \mathrm{~nm}$, respectively. Antioxidantrelated parameter detection kits (SOD A001-1, GSH-Px A005 and CAT A0071) were purchased from Nanjing Jiancheng Bioengineering Institute (Nanjing, China). One unit of SOD activity was defined as the amount of enzyme necessary to produce a $50 \%$ inhibition of the nitro blue tetrazolium reduction rate measured at $550 \mathrm{~nm}$. One unit of GSH-Px activity was defined as the amount of enzyme that reduced the GSH concentration in the reaction system at $1 \mathrm{mmoll}^{-1} \mathrm{~min}^{-1}$. One unit of CAT activity was defined as the amount of enzyme that catalyzed the decomposition of $1 \mathrm{mmol}$ of $\mathrm{H}_{2} \mathrm{O}_{2}$ per min.

\section{Statistical analyses}

White blood cell (WBC), red blood cell (RBC) and platelet values were expressed as the percentage changes compared with the basal value by using one-factor, one-repeated measures of variance (ANOVA), followed by Fisher's least significant difference. The means of the enzymatic and MDA and NO compared with the control group. Significance level was set at $P<0.05$. SPSS 19.0 software was used for all statistical analyses.

\section{RESULTS}

During the experimental periods, no rats treated with linezolid or saline died. The consumption of water and feed was generally lower in three treatment groups, compared with controls, but did not reach statistical significance. Otherwise, the treated rats did not show any other drug-induced physical side effects. parameters of linezolid groups were expressed as the percentage changes

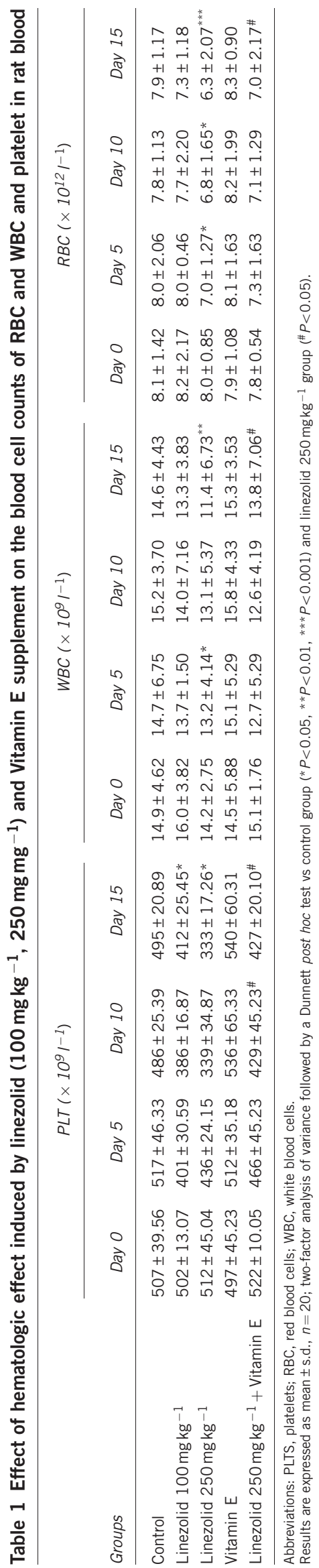

The Journal of Antibiotics 
The blood cell counts (RBC, WBC and platelet) were determined to evaluate the hematological toxicity of linezolid in rats. As shown in Table 1, Vitamin E had no effects on WBC, RBC and platelet numbers compared with control group. Linezolid (100 and $250 \mathrm{mg} \mathrm{kg}^{-1}$ per day) induced a downtrend of $\mathrm{WBC}, \mathrm{RBC}$ and platelet numbers in rat blood with a dose- and time-dependent manner, and this supression of linezolid was partially blocked by the treatment of Vitamin E.

Given the absence of corresponding reports, the present study also examined the effects of linezolid on the oxidative stress and antioxidative capacity in rats. As shown in Figure 1, enzymatic
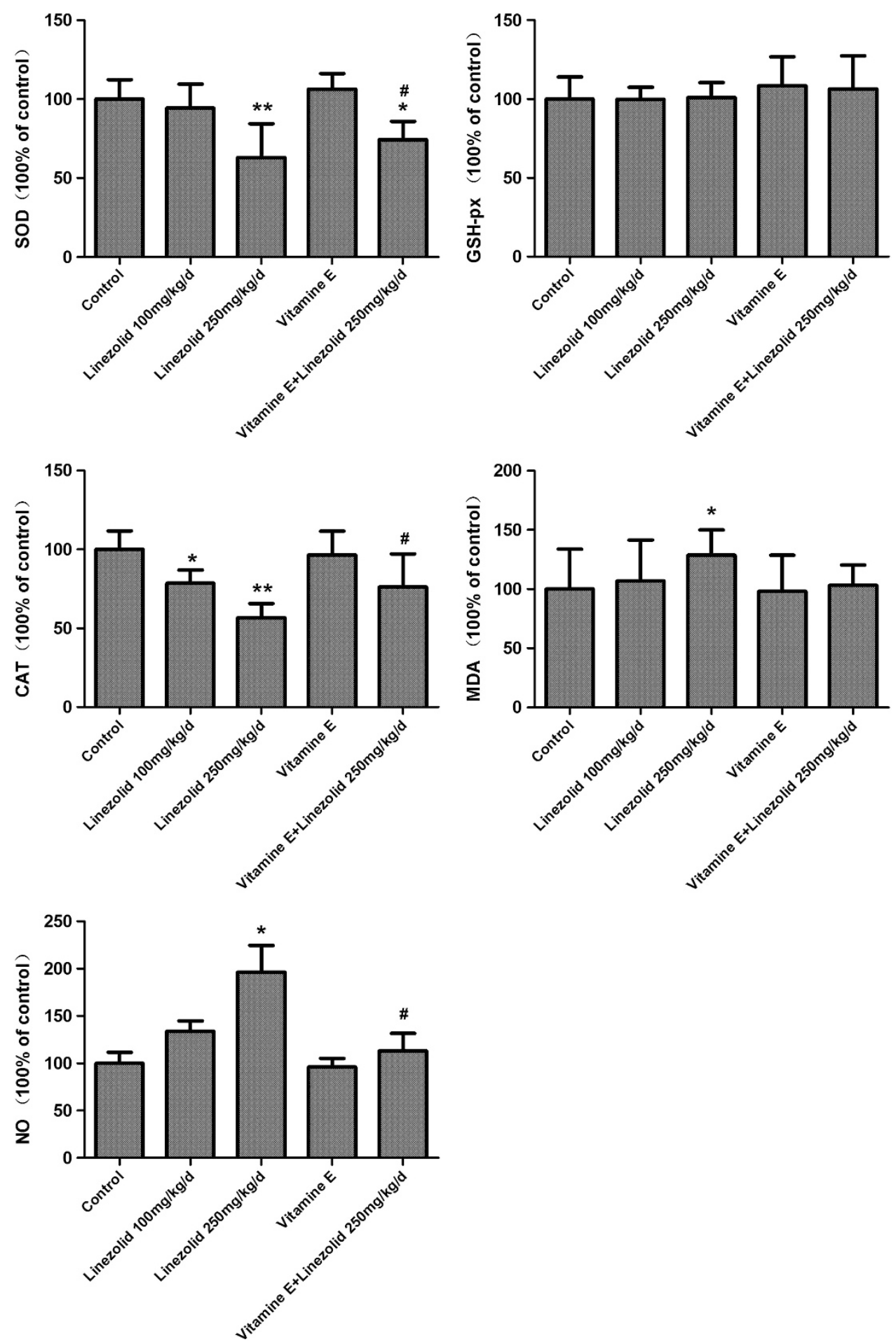

Figure 1 Effect of oxidative stress induced by linezolid ( $100 \mathrm{mg} \mathrm{kg}^{-1}, 250 \mathrm{mg} \mathrm{mg}^{-1}$ ) and Vitamin E supplement on the activity of SOD, GSH-px and CAT, levels of MDA and NO in rat serum. Results are expressed as percentage changes (mean \pm s.e.m.) from basal value (Control group), $n=20$; two-factor

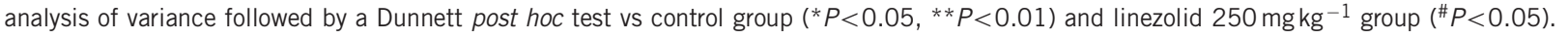


activities of SOD and CAT in rat blood serum showed a dosedependent decrease compared with control group; while the content of MDA and NO was significantly increased in rats serum compared with control group, which was indicative of aggravating oxidative stress and subsequent lipid peroxidation in rats with linezolid. It should be noted that in all three groups linezolid exhibited no significant effect on GSH-Px activities.

Vitamin E supplement did not affect the basal levels of hematological and oxidative parameters in rats, while it partially antagonized the above changes induced by linezolid significantly.

\section{DISCUSSION}

In humans, the approved dosage of linezolid (600 mg op b.i.d.) will achieve a mean ( \pm s.d.) peak steady-state serum level of $21.2 \pm 5.78 \mathrm{mg} \mathrm{ml}^{-1}$, which is intermediate between the mean peak levels of $50 \mathrm{mg} \mathrm{kg}^{-1}$ per day (op) and $125 \mathrm{mg} \mathrm{kg}^{-1}$ per day (op) in rats. ${ }^{16}$ Therefore, in the present study, conventional dose ( $100 \mathrm{mg} \mathrm{kg}^{-1}$ per day) was used to simulate the mean ( \pm s.d.) peak steady-state serum level of linezolid in clinical treatment. Besides that, linezolid was reported to be well tolerated at oral doses of up to $400 \mathrm{mg}$ given three times per day in humans, ${ }^{17}$ so higher dose (250 $\mathrm{mg} \mathrm{kg}^{-1}$ per day) was chosen for observable hematologic effects of linezolid in rats.

There are two major explanations for linezolid-associated hematocytopenia: myelosuppression and immune-mediated mechanism. Waldrep et al. ${ }^{18}$ demonstrated that iron saturation increased in the bone marrow of patients who received linezolid, whereas bone marrow examination in two patients revealed erythroid aplasia and vacuolated erythroblasts, which are classic myelosuppression findings. However, Bernstein et al. ${ }^{19}$ reported an immune-based mechanism for linezolid-induced thrombocytopenia due to the presence of adequate, normal-appearing megakaryocytes in the case. Although the cause and mechanisms of peripheral blood cell decrease are not very clear, it is suggested that this decrease is a result of some immunologic events depending on antibody activities.

In our present research, linezolid can decrease blood cell count (RBC, WBC and platelet) and enzymatic activities of SOD and CAT in a time- and dose-dependent manner. While a significant increase in the MDA and NO levels was observed simultaneously in blood serum of rats. It is illustrated that blood cell lipid peroxidation was enhanced by linezolid, and the activities of SOD and CAT and the inhibition of . $\mathrm{OH}$ were weakened. Under the light of this information, we suggested that oxidative stress effect can be considered among reasons and mechanisms of linezolid-associated hematological effect. Our research also provided the first in vivo evidence that oxidative stress may have an important role in the structural and functional damages of hemocytes.

It is well known that Vitamin $\mathrm{E}$ form the mainstay for effective treatment of antioxidant via preventing the propagation of free radicals in tissues, reacting with them to form a tocopheryl radical as a peroxyl radical scavenger. ${ }^{20}$ In the present study, the hematocytopenia and decrease of antioxidative activity induced by linezolid could be partly recovered by Vitamin E, while Vitamin E per se had no obvious effect on the basic levels of hematological and oxidative parameters. These results suggest that linezolid may have a tonic role, mainly via increasing the number of free radical to decrease antioxidative activity, then induce hematological toxicity.

It is worthwhile to note that GSH-Px activities were relatively stable through the trial course. We all know that CAT and GSH-Px are major primary antioxidant defense component that works to catalyze the decomposition of $\mathrm{H}_{2} \mathrm{O}_{2}$ to $\mathrm{H}_{2} \mathrm{O}$. Previous studies demonstrated that in the presence of low $\mathrm{H}_{2} \mathrm{O}_{2}$ levels, organic peroxides are the preferred substrate for GSH-Px. At high $\mathrm{H}_{2} \mathrm{O}_{2}$ concentrations, they are metabolized by CAT. ${ }^{21}$ SOD could constitute a good molecularbioindicator for acute oxidative stress. ${ }^{22}$ In this regard, we therefore hypothesized that great amount of $\mathrm{H}_{2} \mathrm{O}_{2}$ formation induced by linezolid may be a major mechanism contributing to stable GSH-px level.

However, the exact mechanisms of action, as well as the pharmacological implications of hematological toxicity induced by linezolid are not yet well understood. The actual relationship between oxidative stress and linezolid-associated hematological toxicity is not clear either. Experimental and clinical studies support the pivotal role played by reactive oxidant species in the mechanism of platelet activation. ${ }^{23}$ Anti-Band 3 binding sites (Band 3 senescent antigen) were reported formed on moderately oxidized erythrocytes and thus stimulate these erythrocytes removed from the circulation. ${ }^{24}$ Antioxidant supplementation in patients treated with chemotherapy and radiotherapy apparently decreased oxidative stress, maintained hemoglobin levels. ${ }^{25}$ There are several critical issues that need to be addressed, including the underlying mechanism of action and the effect of antioxidant use on hematological toxicity induced by linezolid.

Under the light of this information, we concluded that the present results first detected the effects of linezolid on basal levels of blood cell in rat serum and revealed the relationship between oxidative stress and hematological toxicity induced by linezolid. The present results might provide a new angle to look for the mechanisms of action of linezolid-associated hematological toxicity.

\section{ACKNOWLEDGEMENTS}

This research is supported by Nursery fund 12KMM42 from the PLA General Hospital. We thank Prof R Wang and P Liu for their technical support.

Author contributions: Tianlin Wang contributed to study design, clinical trial and writing of this study. Daihong Guo contributed to study design. Xianzhe Dong and Lihua Mu contributed to the trial.

1 Yunis, A. A. Chloramphenicol-induced bone marrow suppression. Semin. Hematol. 10, 225-234 (1973)

2 Ambekar, C. S. et al. Metabolism of chloramphenicol succinate in human bone marrow. Eur. J. Clin. Pharmacol. 56, 405-409 (2000)

3 Neu, H. C. Safety of cefepime: a new extended-spectrum parenteral cephalosporin Am. J. Med 100, 68S-75S (1996).

4 Rousan, T. A. et al. Recurrent acute thrombocytopenia in the hospitalized patient: sepsis, DIC, HIT, or antibiotic-induced thrombocytopenia. Am. J. Hematol. 85, 71-74 (2010)

5 DeLougher, T. Drug-induced immune hematologic disease. Immunol Allergy. Clin. North. Am. 18, 830-841 (1998)

6 Yunis, A. A. Chloramphenicol toxicity: 25 years of research. Am. J. Med. 87, 44N-48N (1989).

7 Neftel, K. A., Hauser, S. P. \& Muller, M. R. Inhibition of granulopoiesis in vivo and in vitro by $\beta$-lactam antibiotics. J. Infect. Dis. 152, 90-98 (1985)

8 Senneville, E. et al. Effectiveness and tolerability of prolonged linezolid treatment for chronic osteomyelitis: a retrospective study. Clin. Ther. 28, 1155-1163 (2006).

9 Falagas, M. E. \& Vardakas, K. Z. Benefit-risk assessment of linezolid for serious Grampositive bacterial infections. Drug Saf. 31, 753-768 (2008).

10 Minson, Q. \& Gentry, C. A. Analysis of linezolid-associated hematologic toxicities in a large veterans affairs medical center. Pharmacotherapy 30, 895-903 (2010).

11 Pascoalinho, D., Vilas, M. J., Coelho, L. \& Moreira, P. Linezolid-related immunemediated severe thrombocytopenia. Int. J. Antimicrob. 37, 88-89 (2011).

12 Tsuji, Y. et al. Thrombocytopenia and anemia caused by a persistent high linezolid concentration in patients with renal dysfunction. J. Infect. Chemother. 17, 70-75 (2011).

13 Soundravally, R., Sankar, P., Bobby, Z. \& Hoti, S. L. Oxidative stress in severe dengue viral infection: association of thrombocytopenia with lipid peroxidation. Platelets 19 , 447-454 (2008)

14 Erel, O., Vural, H., Aksoy, N., Aslan, G. \& Ulukanligil, M. Oxidative stress of platelets and thrombocytopenia in patients with vivax malaria. Clin. Biochem. 34, 341-344 (2001) 
15 Ohkawa, H., Ohishi, N. \& Yagi, K. Assay for lipid peroxides in animal tissues by thiobarbituric acid reaction. Anal. Biochem. 95, 351-358 (1979).

16 Slatter, J. G. et al. Pharmacokinetics, toxicokinetics, distribution, metabolism and excretion of linezolid in mouse, rat and dog. Xenobiotica 32, 907-924 (2002)

17 Zurenko, G. E., Ford, C. W., Hutchinson, D. K., Brickner, S. J. \& Barbachyn, M. R. Oxazolidinone antibacterial agents: development of the clinical candidates eperezolid and linezolid. Expert Opin. Investig. Drugs 6, 151-158 (1997).

18 Waldrep, T. W. \& Skiest, D. J. Linezolid-induced anemia and thrombocytopenia. Pharmacotherapy 22, 109-112 (2002).

19 Bernstein, W. B., Trotta, R. F., Rector, J. T., Tjaden, J. A. \& Barile, A. J. Mechanisms for linezolid-induced anemia and thrombocytopenia. Ann. Pharmacother. 37, 517-520 (2003).

20 Traber, M. G. \& Stevens, J. F. Vitamins $C$ and E: beneficial effects from a mechanistic perspective. Free Radic. Biol. Med. 51, 1000-1013 (2011).
21 Yu, B. P. Cellular defenses against damage from reactive oxygen species. Physiol. Rev. 74, 139-162 (1994).

22 Biagini, G., Sala, D. \& Zini, I. Diethyldithiocarbamate, a superoxide dismutase inhibitor, counteracts the maturation of ischemic-like lesions caused by endothelin-1 intrastriatal injection. Neurosci. Lett. 190, 212-216 (1995).

23 Iuliano, L., Colavita, A. R., Leo, R., Praticò, D. \& Violi, F. Oxygen free radicals and platelet activation. Free Radic. Biol. Med. 22, 999-1006 (1997).

24 Beppu, M., Mizukami, A., Nagoya, M. \& Kikugawa, K. Binding of anti-band 3 autoantibody to oxidatively damaged erythrocytes. Formation of senescent antigen on erythrocyte surface by an oxidative mechanism. J. Biol. Chem. 265, 3226-3233 (1990).

25 Fuchs-Tarlovsky, V., Rivera, M. A., Altamirano, K. A., Lopez-Alvarenga, J. C. \& Ceballos-Reyes, G. M. Antioxidant supplementation has a positive effect on oxidative stress and hematological toxicity during oncology treatment in cervical cancer patients. Support Care. Cancer 21, 1359-1363 (2013). 